\title{
Simulação "Movimentos Corpusculares": dos Estados Físicos da Matéria à Mobilidade e Agregação Corpuscular
}

\author{
Carla Morais*
}

\begin{abstract}
$D$ esignados frequentemente por "geração net", "nativos digitais" ou ainda "geração zap", os alunos que se encontram actualmente a frequentar as nossas escolas estão habituados à "acção". Programam o seu tempo para as mais diversas actividades, algumas das quais realizam em simultâneo "clicando" aqui e ali indiscriminadamente. A sua atitude não é diferente quando se encontram perante um recurso educativo digital. Tal facto coloca ao professor, enquanto responsável pela forma como os alunos exploram os recursos educativos que se lhes apresentam, um desafio que urge atender - travar os "cliques" sucessivos contrariando a atitude pedagogicamente pouco profícua de "varrer" o programa educativo rápido de mais sem dele retirar o proveito desejável. Neste sentido, os roteiros de exploração podem constituir-se como importantes instrumentos de potenciação pedagógica de recursos educativos ao fazerem a ponte entre estes recursos e os elementos que os contextualizem em situações e objectivos pedagógicos concretos. É fundamental fornecer aos alunos "dicas" de natureza operacional, intercaladas com questões de natureza interpretativa e reflexiva para que o caminho percorrido, embora desejavelmente personalizado e construído pelo próprio, seja gerador de mais aprendizagens significativas. No presente artigo, para além de algumas breves considerações sobre a estrutura e utilização de roteiros de exploração, apresenta-se a simulação "Movimentos corpusculares", bem como um possível roteiro de exploração da mesma.
\end{abstract}

\section{1) Roteiros de Exploração: víNCU- LOS ENTRE OS RECURSOS DIGITAIS E OS OBJECTIVOS PEDAGÓGICOS}

Tendo como principal objectivo estreitar a relação entre os recursos educativos digitais e os objectivos de aprendizagem que se pretendem desenvolver, os roteiros de exploração deverão ser meios para fomentar nos alunos - que nasceram e cresceram na era digital, que são tipicamente "activos" e em simultâneo fazem zapping entre as dezenas de canais de televisão, trocam SMS constantemente, vão para o computador, onde acedem ao e-mail, navegam na Internet, comunicam online e participam nas redes sociais [1, 2] - o gosto pela pesquisa, pela reflexão, pela participação activa na construção do conhecimento e, acima de tudo, pelo acto de aprender. Tipicamente a estrutura dos roteiros de exploração é

Faculdade de Ciências da Universidade do Porto Centro de Física Computacional da Universidade de Coimbra

E-mail: carlamorais@imediato.pt composta por um conjunto de "dicas" de natureza operacional (muitas vezes acompanhadas por print-screens que ajudem na transição entre o recurso educativo e o roteiro de exploração), misturadas com questões de cariz interpretativo e outras de cariz reflexivo. Uma das nuances associadas à construção dos roteiros poderá passar por conseguir encontrar o ponto de equilíbrio entre a liberdade construtivista e a mínima orientação, permitindo aos alunos uma exploração mais enriquecedora dos recursos educativos digitais [3, 4]. Durante a interacção dos alunos com estes recursos, mediada pela utilização de um roteiro de exploração, o professor deverá procurar [5]:

Explicar claramente o objectivo e a estrutura geral deste instrumento.

$\checkmark$ Ser o menos interventivo possível, embora seja desejável que esteja presente e, se necessário, ajude os alunos.

$\checkmark$ Estar atento a grupos menos acti- vos, procedendo a algum estímulo ou questão que impulsione o trabalho.

$\checkmark$ "Vigiar" se há alunos a correr o recurso educativo digital rápido de mais.

$\checkmark$ Estar à vontade na "navegação" do próprio recurso.

$\checkmark$ Solicitar aos alunos que tomem nota das respostas e dos registos pedidos ao longo do roteiro de exploração, numa folha de papel ou num ficheiro de processamento de texto.

\section{2) Simulação "Movimentos Corpusculares"}

Ainda que de uma forma necessariamente breve, parece-nos pertinente referir que a utilização de simulações computacionais no ensino poderá contribuir para:

Despertar ou aumentar o interesse dos alunos.

$\checkmark$ Promover uma abstracção facilitada levando a uma compreensão mais sustentada dos conceitos químicos. 
Desenvolver hipóteses, testá-las, analisar resultados e aperfeiçoar os conceitos.

$\checkmark$ Criar um ambiente interactivo, "hands-on", de aprender fazendo.

Além dos aspectos enunciados, cabenos realçar que a utilização de simulações computacionais no ensino é coerente com a própria prática de investigação científica, que, cada vez mais as utiliza [6 - 11].

Para que possamos ficar com uma visão global do recurso educativo "Movimentos corpusculares", cujo roteiro de exploração se encontra em anexo a este artigo, apresentamos de seguida algumas das suas principais características:

- Tipologia do recurso: simulação computacional.

- Disponível em: "Manual Multimédia 8CFQ" publicado, em 2007, pela Texto Editores [12]. O "Manual Multimédia" é um produto inovador, conjugando num único CD-ROM o livro escolar [13], em formato digital, com múltiplos recursos multimédia interactivos que vão desde simulações, jogos, vídeos, áudios e animações até avaliações e módulos de ampliação de conhecimentos (Figura 1).

- Público-alvo: alunos do $8 .^{\circ}$ ano de escolaridade da disciplina de Ciências Físico-Químicas.

- Temática de integração do recurso: estados físicos da matéria e agregação corpuscular.

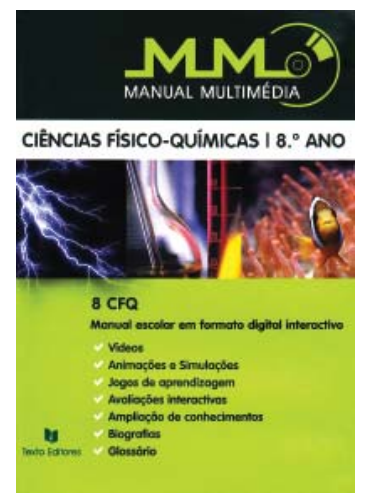

Figura 1 - "Manual Multimédia 8CFQ", Texto Editores [12]

- Metas de aprendizagem previstas:

$\rightarrow \rightarrow$ Compreensão de que a matéria é constituída por corpúscu- los em constante movimento.

$\rightarrow$ Reconhecimento de que os corpúsculos de uma substância se movem incessantemente, quer se trate de substâncias sólidas, líquidas ou gasosas.

$\rightarrow$ Distinção dos estados físicos da matéria em termos de agregação corpuscular.

$\rightarrow$ Reconhecimento do carácter mais limitado dos movimentos corpusculares nos sólidos e nos líquidos e menos nos gases.

$\cdots$ Associação da variação da temperatura de uma substância com a variação da velocidade média dos seus corpúsculos constituintes.

$\rightarrow \rightarrow$ Relação entre estado físico e organização corpuscular e as propriedades dos materiais.

- Breve descrição do recurso educativo digital:

A simulação computacional iniciase com a apresentação de uma breve contextualização teórica, seguindo-se um novo ecrã no qual está representada a substância água no estado sólido (cubos de gelo), no estado líquido (um copo com água) e no estado gasoso (vapor de água que sai do interior de uma panela que está exposta à chama). Perante estas opções, o aluno deverá seleccionar a água num dos três estados físicos apresentados. Uma vez feita esta selecção, aparece a imagem ampliada da água no estado físico seleccionado e de seguida surge um instrumento imaginário de zoom activo, cujo objectivo é marcar claramente que a partir daquele momento se iniciou uma incursão no mundo microscópico (zoom in), para explorar a relação entre o estado físico da água e a respectiva agregação corpuscular. Perante a representação simbólica dos corpúsculos, o aluno tem a possibilidade de simular variações de temperatura deslocando a barra vermelha do termómetro e verificar quais são as implicações de tais alterações na velocidade média dos corpúsculos. De seguida poderá regressar ao ecrã de selecção e escolher a água num outro estado físico (Figura 2).
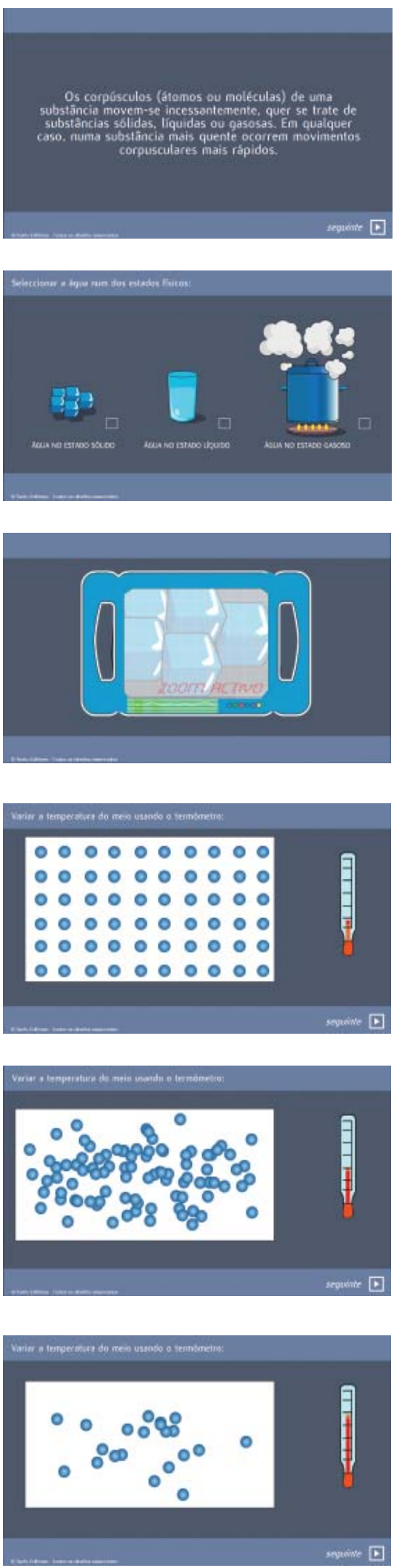

Figura 2 - Vários ecrãs da simulação "Movimentos corpusculares"

$\mathrm{Na}$ senda de outros artigos já publicados no boletim da SPQ [3 - 5], o roteiro de exploração que propomos segue as linhas de força apontadas para estes instrumentos de potenciação pedagógica de recursos digitais 
e utiliza uma linguagem simples e adequada aos alunos, tendo sempre presente o rigor científico. No âmbito de um projecto de investigação, está a ser avaliado o impacto da utilização da simulação e deste roteiro de exploração, junto dos alunos de três turmas do $8 .^{\circ}$ ano de escolaridade. Os alunos de todas as turmas começaram por realizar um pré-teste, que incluía questões alusivas aos estados físicos da matéria e à mobilidade e agregação corpuscular. De seguida, em duas das turmas, está a decorrer a aplicação do programa de intervenção, constituindo-se a terceira turma como grupo de controlo. $\mathrm{Na}$ fase final do estudo, os alunos de todas as turmas realizarão um pós-teste equivalente ao pré-teste inicial. Como técnicas de recolha de dados, para além da técnica de inquérito por questionário (pré-teste, roteiro de exploração e pós-teste) irão ser usadas outras técnicas como a observação e o inquérito por entrevista.

\section{Bibliografia}

[1] C. Morais. + Química Digital - Recursos digitais no ensino da Química: uma experiência no $7 .^{\circ}$ ano de escolaridade.
Dissertação de Mestrado em Educação Multimédia, Faculdade de Ciências da Universidade do Porto, Porto, Portugal (2006).

[2] Learning to Change: ICT in Schools. OECD - organization for economic cooperation and development. Schooling for Tomorrow. Paris (2001) Disponível em http://www.eric.ed.gov/ ERICWebPortal/contentdelivery/ servlet/ERICServlet?accno=ED459698

[3] C. Morais, J. C. Paiva. Roteiros de Exploração - Usando o programa sobre equilíbrio químico "Le Chat". Boletim da Sociedade Portuguesa de Química 100 (2006) 87-89.

[4] J. C. Paiva, L. A. Costa. Roteiros de Exploração - valorização pedagógica de software educativo de Química. Boletim da Sociedade Portuguesa de Química 96 (2005) 64-66.

[5] F. B. Ferreira, J. C. Paiva. Roteiros de exploração com Tabelas Periódicas digitais. Boletim da Sociedade Portuguesa de Química 96 (2005) 67-68.

[6] C. Morais, J. C. Paiva. Simulação digital e actividades experimentais em Físico-Químicas. Estudo piloto sobre o impacto do recurso "Ponto de fusão e ponto de ebulição" no $7 .^{\circ}$ ano de escolaridade. Sísifo. Revista de Ciências da Educação, 3, (2007) 101-111.
[7] L. Chodroff, T. M. O'Neal, D. A. Long, S. Hemkin. An Educational Approach to Computationally Modeling Dynamical Systems. Journal of Chemical Education 86 (2009) 1072-1076.

[8] P. R. Burkholder, G. H. Purser, R. S. Cole. Using Molecular Dynamics Simulation to Reinforce Student Understanding of Intermolecular Forces. Journal of Chemical Education 85 (2008) 1071.

[9] V. Gil, J. C. Paiva. Computer Simulations of Salt Solubility. Journal of Chemical Education 83 (2006) 173-174 .

[10]V. Gil, J. C. Paiva. Using Computer Simulations to Teach Salt Solubility. The Role of Entropy in Solubility Equilibrium. Journal of Chemical Education 83 (2006) 170-172.

[11] J. C. Paiva, V. Gil, A. Ferrer Correia. Le Chat: simulation in Chemical Equilibrium. Journal of Chemical Education 79 (2002) 640-641.

[12] C. Fiolhais; M. Fiolhais; V. Gil; J. Paiva; C. Morais; S. Costa. Manual Multimédia 8CFQ. Texto Editores, Lisboa (2007).

[12] C. Fiolhais; M. Fiolhais; V. Gil; J. Paiva; C. Morais; S. Costa. 8CFQ- Ciências Físico-Químicas. Texto Editores, Lisboa (2007).

\section{Aciualidade Científica}

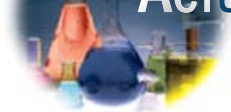

\section{Nanopartículas de PRATA: A ChAVE PARA A CONFIRMAÇÃo DE UM LEITE SEM MELAMINA}

O número alarmante de crianças chinesas vítimas em 2008 da ingestão de leite contaminado com melamina suscitou, no país, a necessidade de melhorar os padrões de detecção de contaminantes químicos nos alimentos. Embora diversas técnicas tenham sido desenvolvidas neste contexto, estas revelaram-se pouco promissoras para determinações rotineiras e efectuadas em campo dado que envolvem equipamento especializado, nomeadamente espectrómetros de massa. Como alternativa, C. Han and $\mathrm{H}$. Li da Universidade Normal da China Central (Wuhan) construíram um sensor de nanopartículas de prata que muda de cor (amarelo para verde escuro) na presença de melamina.
Desta forma, com resultado visto a olho nu, este método colorimétrico altamente sensível não só vem facilitar a detecção da melamina como também assegurar a sua portabilidade, podendo ser utilizado inclusivamente para uso doméstico. As nanopartículas de prata são modificadas com para-nitroanilina ( $p$-NA) e são facilmente preparadas utilizando materiais disponíveis no mercado. Da interacção electrónica dador-aceitador entre a melamina (dador) e a $p$-NA (aceitador) resulta a agregação das nanopartículas de prata e, como consequência, ocorre mudança de cor. A detecção da melamina é, deste modo, conseguida ao fim de apenas 2 minutos sendo possível detectar até $0.1 \mathrm{mg} / \mathrm{L}$ na amostra.
Para além das nanopartículas de prata, também as de ouro já tinham sido inicialmente testadas para este fim, mas os resultados não foram adequados à sua disseminação. Tal como reportam os investigadores, estas exigem um estabilizador adicional para detectar a melamina que é difícil de sintetizar e que limita a sua aplicação prática. O próximo passo será facilitar o uso do sensor pelo público geral nas suas casas tentando, assim, disponibilizá-lo sob a forma de tira de teste.

(Fonte: http://www.rsc.org/ Publishing/ChemScience/ Volume/2010/03/safer_milk.asp) JNR 


\title{
XVI Encontro \\ Luso-Galego de Química
}

\author{
Universidade de Aveiro \\ Aveiro, Portugal
}

10 a 12 de Novembro de 2010

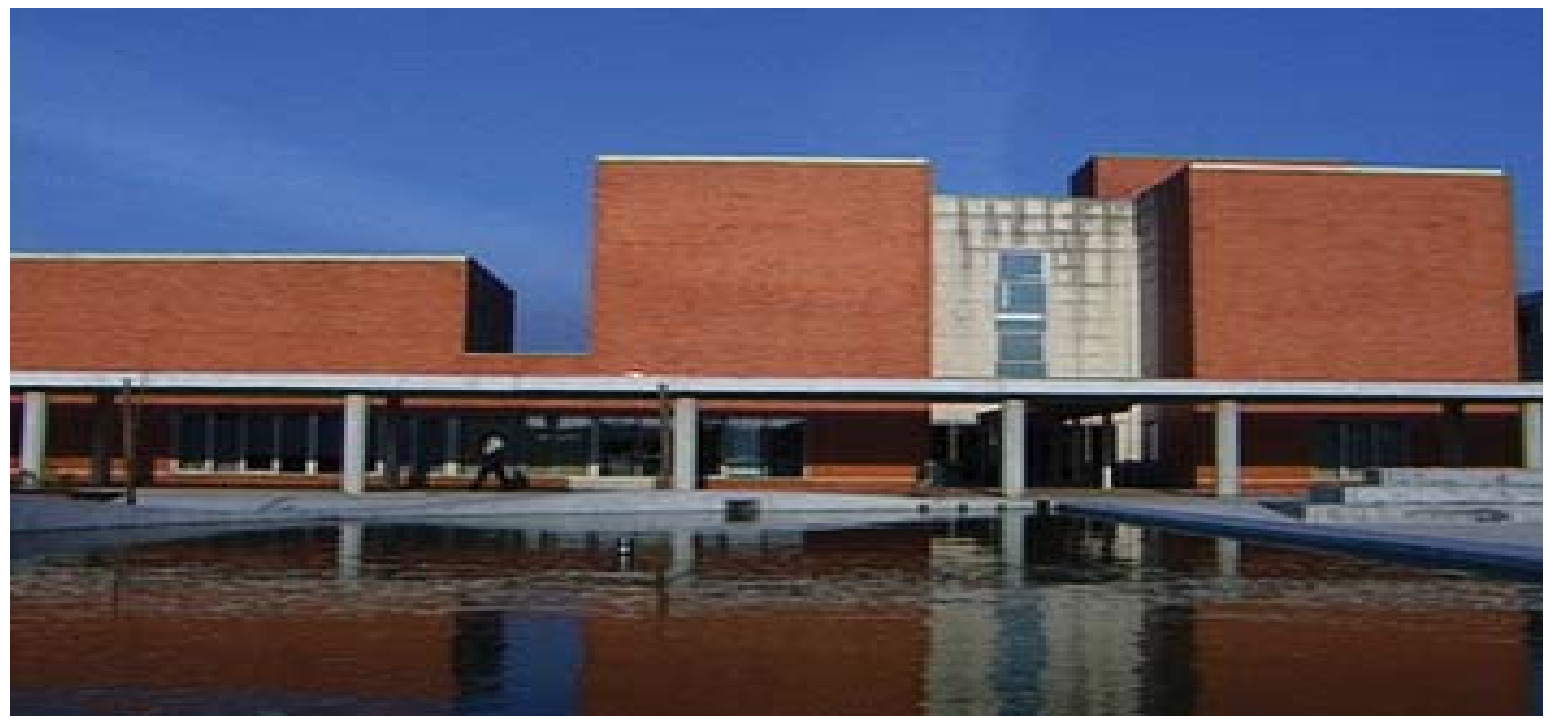

\section{LIÇÕES PLENÁRIAS}

ARMando Pombeiro (Departamento de Engenharia Química, Instituto Superior Técnico, Universidade Técnica de Lisboa) - "Funcionalização de alcanos em condições suaves"

JosÉ TeIXeIRA (Departamento de Engenharia Biológica, Escola de Engenharia da Universidade do Minho) - “A química e a biotecnologia: uma parceria de sucesso"

RENÉ SANTUS (Départament RDDM, Muséum National d'Histoire Naturelle, Paris) - “Unravelling the mechanisms of reactions of natural and synthetic antioxidants in biologically relevant multiphasic aqueous systems by pulse radiolysis"

María Tarsy Carballas Fernández (Instituto de Investigaciones Agrobiológicas de Galiza, CSIC, Santiago de Compostela) - "Repercussão dos incêndios florestais na qualidade do solo"

Manuel López-Rivadulla Lamas (Instituto de medicina Legal, Facultat de Medicina, Universidad de Santiago de Compostela) - "Drogas e condução"

\section{DATAS IMPORTANTES}

DATA LIMITE PARA SUBMISSÃO DE RESUMOS:

18/Outubro/2010

CONFIRMAÇÃO DA ACEITAÇÃO DAS COMUNICAÇÕES:

25/Outubro/2010

DATA LIMITE PARA INSCRIÇÃO NO ENCONTRO:

28/Outubro/2010

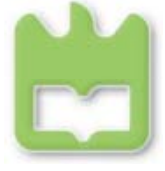

Universidade de Aveiro

Colegio Oficial de Químicos de Galicia

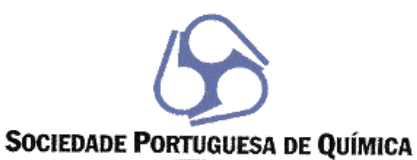

SOCIEDAde PoRTuguesa de Química
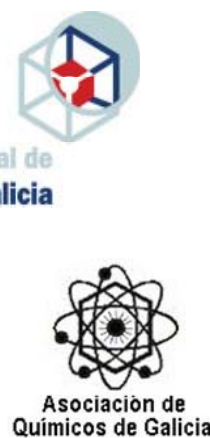
Ícones simbólicos utilizados no roteiro de exploração e respectivo significado:

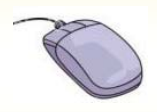

"Dicas" operacionais

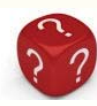

Questões

\section{Questões de salto}

O professor poderá dispensar os alunos de responderem a estas questões, se assim o entender ou se as questões imediatamente anteriores tiverem sido respondidas correctamente.
1. Uma vez iniciada a exploração da simulação educativa, surge um pequeno texto introdutório com informações importantes sobre a temática que será abordada neste recurso. Lê com atenção esse texto.

2. Clica no botão "seguinte" para continuares.

3. Neste novo ecrã tens a possibilidade de seleccionar a água num dos três estados físicos apresentados.

4. Começa por seleccionar a água no estado sólido no correspondente.

5. Clica no botão "seguinte" para continuares e observa com atenção a animação que surge no novo ecrã.

6. Atende à imagem ampliada da água no estado sólido e ao aparecimento de um instrumento imaginário de zoom activo.

\section{Q1:}

Sabendo que um dos propósitos desta simulação é explorar a relação entre o estado físico de uma substância (como por exemplo a água) e a respectiva agregação corpuscular, explica qual será o objectivo de utilizar este instrumento de zoom activo.

7. Observa a organização e os movimentos corpusculares da substância água no estado sólido.

\section{Q2:}

Quando uma substância se encontra no estado sólido, como a água neste exemplo, o que se verifica relativamente:

a) Ao movimento dos seus corpúsculos?
b) À proximidade e à agregação entre esses corpúsculos?

Q3:

A proximidade e a agregação entre os corpúsculos influenciam as propriedades dos materiais. Tendo em conta o grau de proximidade e de agregação entre os corpúsculos dos materiais no estado sólido, estes materiais apresentam:

(Assinala a opção correcta)
Forma e volume variáveis.

Forma e volume constantes (a temperatura constante).

Volume constante (a temperatura constante) mas forma variável.

\section{Q4:}

Quando se aumentar a temperatura da água, prevês alguma alteração no comportamento dos seus corpúsculos constituintes?

(Assinala a opção correcta)

Sim.

Não.

Q5: Se na questão anterior respondeste: *

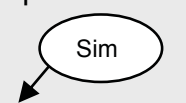

Procura explicar qual é a alteração que prevês.

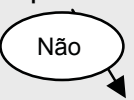

Avança para o ponto 8 do roteiro.
8. Colocando o cursor do rato sobre o indicador do termómetro, faz variar a temperatura.

\section{Q6:}

Explica o que aconteceu aos corpúsculos quando se aumentou a temperatura.

Q7:

Explica o que aconteceu aos corpúsculos quando se diminuiu a temperatura. *

9. Clica no botão "seguinte" para regressares ao ecrã inicial.

10. Selecciona agora a água no estado líquido.

11. Clica no botão "seguinte" para continuares e observa com atenção a animação que surge no novo ecrã.

12. Atende à organização e aos movimentos corpusculares da substância água no estado líquido.

\section{Q8:}

Quando uma substância se encontra no estado líquido, como a água neste exemplo, o que se verifica relativamente: 
a) Ao movimento dos seus corpúsculos?

b) Aos espaços vazios existentes entre esses corpúsculos?

Q9:

Tendo em conta o grau de proximidade e de agregação entre os corpúsculos dos materiais no estado líquido, estes materiais apresentam:

(Assinala a opção correcta)

Forma e volume variáveis.

Forma e volume constantes (a temperatura constante)

Volume constante (a temperatura constante) mas forma variável.

\section{Q10:}

Quando se aumentar a temperatura da água, prevês que, à semelhança do que aconteceu com a água no estado sólido, também aqui no estado líquido se verifique alguma alteração no comportamento dos corpúsculos?

(Assinala a opção correcta) *

Sim.

Não.

13. Colocando o cursor do rato sobre o indicador do termómetro, faz variar a temperatura.

Q11:

Explica o que aconteceu aos corpúsculos quando se aumentou a temperatura. *

Q12:

Explica o que aconteceu aos corpúsculos quando se diminuiu a temperatura.

Q13:

Estabelecendo uma análise comparativa, o que se pode dizer relativamente à liberdade de movimento dos corpúsculos da água no estado líquido e no estado sólido? *

14. Clica no botão "seguinte" para regressares ao ecrã inicial.

15. Selecciona, por último, a água no estado gasoso.

16. Clica no botão "seguinte" para continuares e observa com atenção a animação que surge no novo ecrã.

17. Atende, particularmente, à organização e aos movimentos corpusculares da substância água no estado gasoso.

\section{Q14:}

Quando uma substância se encontra no estado gasoso, como a água neste exemplo, o que se verifica relativamente: a) Ao movimento dos seus corpúsculos?

b) À proximidade e à agregação entre esses corpúsculos?

\section{Q15:}

Tendo em conta o grau de proximidade e de agregação entre os corpúsculos dos materiais no estado gasoso, estes materiais apresentam:

(Assinala a opção correcta)

Forma e volume variáveis.

Volume constante (a temperatura constante) mas forma variável.

Forma e volume constantes (a temperatura constante.

18. Colocando o cursor do rato sobre o indicador do termómetro, faz variar a temperatura.

\section{Q16:}

Em qual dos três estados físicos os corpúsculos estão menos agregados e apresentam maior liberdade de movimento?

(Assinala a opção correcta)

Sólido.

Líquido.

Gasoso.

\section{Q17:}

Em qual dos três estados físicos os corpúsculos estão mais agregados e apresentam menor liberdade de movimento?

(Assinala a opção correcta) *

Sólido.

Líquido.

Gasoso.

Q18:

Completa correctamente as frases:

Embora haja diferenças na maneira como os corpúsculos estão associados uns aos outros, os corpúsculos de uma substância movem-se

....quer se trate de uma substância sólida , ..... ou ... Em qualquer caso, numa substância mais .... ocorrem movimentos corpusculares ...... rápidos.

\section{1ting}

1il Pesquisa na Internet informações que te ajudem a explicar o facto de um sólido poder ter uma temperatura mais alta do que um gás, apesar de os corpúsculos do sólido terem movimentos bastante mais restritos. Elabora e regista alguns tópicos resultantes desta pesquisa. 